\title{
Zernike Cryo-EM with a Direct Electron Camera Enables Tracking Protein Conformations in the Temporal Dimension
}

$\underline{\text { Yi-min }} \mathrm{Wu}^{1}$, Jen-wei Chang ${ }^{2}$, Chun-hsiung Wang ${ }^{3}$, Kuniaki Nagayama ${ }^{4}{ }^{*}$, Naoyuki Miyazaki ${ }^{5}$, Kazuyoshi Murata $^{6}$ and Wei-hau Chang ${ }^{7}$

1. Institute of Chemistry, Academia Sinica, Taipei Taiwan 115.

2. Institute of Chemistry, Academia Sinica, Taipei Taiwan 115

3. Institute of Chemistry, Academia Sinica, Taipei Taiwan 115

4. National Institute for Physiological Sciences, Okazaki, Aichi, 444-8585, Japan

5. National Institute for Physiological Sciences, Okazaki, Aichi, 444-8585, Japan

6. National Institute for Physiological Sciences, Okazaki, Aichi, 444-8585, Japan

7. Institute of Chemistry, Institute of Physics and Genomic Research Center, Academia Sinica, Taipei

Taiwan 115

* Current Address: Sokendai, Hayamacho, Kanagawa, 240-0193, Japan

Direct electron camera built on CMOS chips with high quantum yield and video frame rate has begun to revolutionize biological cryo-electron microscopy (cryo-EM). By recording a molecular image as frames instead of an accumulated exposure, post-imaging computation are available, by which the chargeinduced or drift-caused image blurring are to be corrected in 2D by aligning the same particle images in the movie. As such, the number of near atomic and that of sub-nanometer structures are rapidly increasing, with notable examples including a mosaic virus [1] and a TRP tetramer channel [2] imaged by $300 \mathrm{kV}$ field emission instruments.

To test how far one can reach using an average $200 \mathrm{kV}$ field emission instrument equipped with a direct electron camera with the above-mentioned motion-correction approach, we studied a DGNNV virus with icosahedral symmetry, $35 \mathrm{~nm}$ in diameter, and reached a near atomic resolution for its capsid in the core region from 15000 single particle images [3]. As we attempted to expand the same approach to asymmetric but smaller protein complex, we resorted to the usage of Zernike phase plate that provides superior contrast than conventional defocusing microscopy [4-5]. To this end, we used a $200 \mathrm{kV}$ field emission instrument at Okazaki equipped with a direct electron camera. We chose RNA polymerase II complex $(\sim 12 \mathrm{~nm})$ as a benchmark molecule as its known atomic structure would help data interpretation and the possibility of revealing it by using Zernike cryo-EM were examined both by simulations [6-7] and by experiment [8]. However, we encountered a $\sim 20 \AA$ resolution barrier for which we summed all the motion-corrected frames as mentioned above to yield an RNA polymerase II structure on which the surface motifs were blurred, suggesting the existence of other type of motions during the imaging period cannot be corrected for in 2D, including orientation change of particles.

To this end, we resorted to Scheres scheme of running averages of 6 movie frames [9], corresponding to 0.25 second, and reached a sub-nanometer resolution structure for RNA polymerase II using 40000 single particle Zernike images. Interestingly, as we relaxed the target resolution for 3D reconstruction of RNA polymerase II to $20 \AA$, we found that the running averages of 3 movie frames would still give enough signals for accurately determining the orientation for particles as small as RNA polymerase II for the signals limited by small number of electrons could be compensated by the high contrast. Remarkably, such the first 3 movie-frame $20 \AA$ RNA polymerase II differs from the one-second accumulated RNA polymerase II in that many surface motifs become recognizable. Based on this 
finding, we went on to fraction the one second into various time points to ask whether or not the 3D reconstruction from the same set of particles would result in the same conformation at different time. To our surprise, significant rearrangement of the RNA polymerase II structure along the time trajectory was observed where most of the notable motions are consistent with those based on the X-ray crystal studies, including the motions of the clamp and the stalk.

[1] Z Wang et al, Nat Commun. 5, p. 4808.

[2] M Liao et al, Nature 504, p. 107-112.

[3] CH Wang and WH Chang, in preparation.

[4] R Danev and K Nagayama, Ultramicroscopy 88, p. 243-52.

[5] W Dai et al, Nature 502, p. 707-710..

[6] WH Chang et al, Structure 18, p. 17-27.

[7] RJ Hall et al, J Struct Biol. 174, p. 468-75.

[8] YM Wu et al, J Physics D: Appl. Phys. 46, p. 494008

[9] A Brown et al, Science 346, p. 718-722

[10] The authors acknowledge funding from the Academia Sinica and NSF in Taiwan and NINS in Japan. 